\title{
Impacto da cultura organizacional no desequilíbrio entre esforço-recompensa no trabalho em uma universidade pública federal
}

Márcia Regina Teixeira Minari ${ }^{1}$, Liliana Andolpho Magalhães Guimarães

Universidade Católica Dom Bosco (UCDB), Campo Grande, Mato Grosso do Sul, Brasil

\section{Palavras-chave:}

Cultura organizacional,

Estresse psicológico,

Universidades,

Pessoal administrativo.

\section{Resumo}

Este estudo investigou o papel da cultura organizacional (CO) em relação ao desequilíbrio entre esforço-recompensa no trabalho em servidores públicos do ensino superior. Foi realizado estudo quantitativo em uma amostra de 302 técnico-administrativos de uma universidade federal. Foram utilizados o Instrumento Brasileiro para Avaliação da Cultura Organizacional (IBACO) e o Questionário de Desequilíbrio entre Esforço-Recompensa no Trabalho (EffortReward Imbalance - ERI). Fatores da CO (profissionalismo cooperativo, satisfação e bem-estar, integração externa, recompensa e treinamento e promoção do relacionamento interpessoal) apresentaram médias significativamente menores no grupo de servidores expostos ao ERI. Na regressão logística, o modelo preditivo da CO, pelo fator satisfação e bem-estar, explicou $42,9 \%$ do ERI. Os resultados encontrados fornecem subsídios empíricos da CO na composição dos riscos à exposição ao estresse ocupacional e indicam sua importância nas ações de promoção, prevenção e intervenção.

Impact of organizational culture on effort-reward imbalance at work at a federal public university

\section{Keywords:}

Organizational culture Psychological stress,

Universities,

Administrative staff
Impacto de la cultura organizacional en el desequilibrio entre esfuerzo-recompensa en el trabajo en una universidad pública federal

\section{Resumen}

Este estudio investigó el papel de la cultura organizacional (CO) con relación al desequilibrio entre esfuerzo-recompensa en el trabajo en servidores públicos de la enseñanza superior. Se utilizó el método cuantitativo con un muestreo de 302 técnico-administrativos de la universidad federal. Los instrumentos utilizados fueron: Instrumento Brasileño para Evaluación de la Cultura Organizacional (IBACO) y Cuestionario de Desequilibrio entre Esfuerzo-Recompensa en el trabajo (Effort-Reward Imbalance - ERI). Factores de la CO (profesionalismo cooperativo, satisfacción y bienestar, integración externa, recompensa y entrenamiento y promoción de la relación interpersonal) presentaron, significativamente, menores porcentajes en el grupo de servidores clasificados como expuestos al ERI. En la regresión logística, el modelo predictivo de la CO, por el factor satisfacción y bienestar, explicó 42,9\% del ERI. Los resultados proporcionan subsidios empíricos acerca de la CO sobre el riesgo de exposición al estrés ocupacional y indican su importância em acciones de promoción, prevención e intervención.

\footnotetext{
Endereço para correspondência:

Av. Tamandaré, 6000 - Jardim Seminário, Campo Grande - MS, 79117-010. Email: martminari@gmail.com

Como citar este artigo:

Minari, M. R. T., \& Guimarães, L. A. M. (2019). Impacto da cultura organizacional no desequilíbrio entre esforço-recompensa no trabalho em uma universidade pública federal. Revista Psicologia: Organizações e Trabalho, 19 (2), 616-623. doi: 10.17652/rpot/2019.2.15580
} 
As universidades, assim como outras instituições públicas ou privadas, também estão imersas nas rápidas transformações do mundo do trabalho, o que as conduzem a enfrentarem os desafios da globalização e as rápidas transformações do conhecimento. Gerações atuais precisam responder rapidamente às mudanças que são contínuas, o que gera a necessidade de ajustes nos estilos de vida, com o intuito de incorporar novos valores e atitudes que emergem desse processo de transformação e que levam a sociedade a revitalizar a vida no trabalho, na família e no pessoal (López, González, Silva, Tolfo \& Cedeño, 2015).

Essas transformações influenciaram, segundo revisão da literatura realizada por Venables e Allender (2006), a saúde ocupacional nas universidades do Reino Unido e a $\mathrm{CO}$, que mudou nas últimas décadas e afetou negativamente a saúde dos trabalhadores. Os autores identificaram ainda que o ensino superior inclui organizações complexas, que contemplam empregadores com COs muito variadas e com altas exposições a riscos psicossociais, como sobrecarga de trabalho, insegurança no trabalho e problemas no controle de recursos e de comunicação organizacional.

O trabalho de Biron, Brun e Ivers (2008) também constatou que as práticas de gestão de recursos humanos não acompanharam as rápidas mudanças organizacionais que afetaram as universidades canadenses nos últimos anos. Os autores, ao analisarem as categorias de docentes e funcionários administrativos, identificaram que $40,9 \%$ dos servidores reportaram elevado nível de sofrimento psicológico. A sobrecarga de trabalho, a relação com os superiores e a baixa participação na tomada de decisão organizacional foram sistematicamente relatadas como elevados riscos para a saúde dos funcionários.

Em estudo realizado no Brasil, Gradella Júnior (2010) concluiu que a organização do trabalho em uma universidade pública revelou-se um ambiente permeado de sofrimento psíquico e alienação. Os resultados dessa pesquisa revelaram que $29,6 \%$ dos servidores docentes reportaram algum problema de saúde após o ingresso na universidade, que relacionaram principalmente a questões de organização e condições inadequadas de trabalho, situações que tendem a prejudicar a realização da tarefa pela produção do conhecimento, como falta de infraestrutura adequada de bibliotecas, laboratórios, material didático-pedagógico, entre outras.

Nesse sentido, torna-se relevante o cuidado com os servidores do ensino superior, uma vez que estes têm alta responsabilidade social para a formação de indivíduos, não só para responder às necessidades do mercado de trabalho, como também para desenvolver a transformação social, política, econômica, técnica e científica a nível local, regional e global (Knudson, 2009). Além da importância do papel dos docentes nesse contexto, priorizou-se, neste estudo, a carreira técnico-administrativa, já que ela contribui para o desenvolvimento dos processos administrativos e funcionamento geral da universidade, em contato direto com usuários internos e externos ao ambiente universitário.

Para identificar as características que perpassam o serviço público federal e quais seus possíveis impactos na saúde mental dos servidores técnico-administrativos do ambiente universitário, este estudo pretendeu ampliar as reflexões sobre a saúde mental, por meio da investigação do risco para estresse ocupacional e sua possível influência na CO. A investigação da CO ocorreu devido a importância de os funcionários melhor entenderem como as organizações funcionam, além de compreenderem as normas e os padrões de comportamento das pessoas na organização para alcançarem metas e objetivos da instituição (Lira, 2015).

Entre as diversas perspectivas teóricas utilizadas para compreender a $\mathrm{CO}$, foi adotado, nesta pesquisa, o modelo teórico de
Hofstede, Neuijen, Ohayv e Sanders (1990), por contemplar tanto a análise das práticas organizacionais, que incluem símbolos (palavras, gestos e objetos que têm significado especial dentro da organização), heróis (personagens vivos e mortos, reais ou imaginários, que servem de modelo de comportamento para seus membros) e rituais (atividades coletivas), quanto o estudo dos valores organizacionais, que se referem a sentimentos raramente discutidos (Ferreira \& Assmar, 2008). Para Hofstede, Hofstede e Minkov (2010), os valores dos líderes e fundadores influenciam e moldam a cultura, porém, é por meio de práticas diárias, transmitidas aos membros mediante mecanismos de socialização, que essa cultura afeta os trabalhadores, razão pela qual as percepções compartilhadas de tais práticas constituem a essência da cultura de uma organização.

Para a análise do risco para estresse ocupacional, foi utilizado o modelo teórico-metodológico denominado Desequilíbrio entre Esforço-Recompensa no Trabalho (Effort-Reward Imbalance - ERI), de Siegrist (1996), selecionado por avaliar o risco psicossocial no trabalho e apresentar boa evidência epidemiológica na análise da saúde mental laboral (Ansoleaga, 2015; Griep, Rotenberg, Landsbergis \& Vasconcellos-Silva, 2011;). O modelo ERI pressupõe que as experiências cronicamente estressantes são decorrentes de desequilíbrio entre alto esforço (pressões no trabalho) e baixa recompensa (salário, segurança e reconhecimento), somados a alto nível pessoal de supercomprometimento no trabalho (Herr et al., 2017; Landolt, O'Donnell, Hazi, Dragano \& Wright, 2017). O modelo também postula que a falta de reciprocidade entre os esforços empreendidos e os retornos obtidos em seu trabalho pode levar os trabalhadores a reações associadas de tensão, com efeitos adversos de longo prazo à saúde (Silva \& Barreto, 2010; Griep et al., 2011).

Destaca-se que, para análise deste estudo, foram contemplados diversos marcadores teóricos e metodológicos advindos da abordagem do Work Stress (Seligmann-Silva, 1995; Jacques, 2003), priorizando não somente o trabalhador de maneira individual, mas principalmente os aspectos sociais referentes ao âmbito da organização de trabalho. Busca, desse modo, a interação dinâmica entre a pessoa e o seu ambiente de trabalho (Glina, 2014). Compartilha-se ainda o entendimento de que o trabalho tem um papel estruturante na vida, na identidade do indivíduo e na própria construção da condição humana e das sociedades. Assim, suas condições precárias ou enriquecedoras e as formas como as pessoas se relacionam com essas condições poderão estar envolvidas tanto no surgimento de enfermidades quanto na valorização e realização do indivíduo (Borges, Guimarães \& Silva, 2013).

\section{Cultura organizacional e estresse ocupacional}

No campo teórico das possíveis influências da CO sobre o estresse ocupacional, Peterson e Wilson (2002) propõem o modelo cultura-trabalho-saúde e estresse no trabalho, que preconiza o papel da CO na etiologia do estresse no local de trabalho. De forma semelhante, Ferreira e Assmar (2004) propuseram um modelo integrado de cultura-satisfação-saúde no trabalho, apoiado em pressupostos de que, quando valores organizacionais, normas e práticas dominantes deixam de lado a preocupação com o trabalhador, potencialmente surgem conflitos e por consequência problemas de saúde, como ansiedade e depressão. Tamayo (2008) identificou, em revisão de literatura, a quase inexistência de pesquisas empíricas a respeito da relação entre a $\mathrm{CO}$, especificamente pelos valores organizacionais, e o estresse ocupacional. Dessa forma, apontou a importância de futuras pesquisas investigarem o impacto dos valores organizacionais, considerados variáveis nucleares da $\mathrm{CO}$, em relação ao estresse ocupacional, para desenvolver uma visão global 
e ampliada do ambiente de trabalho sobre a saúde do trabalhador. Também Dextras-Gauthier, Marchand e Haines (2012) sugeriram uma análise estrutural que relacione a $\mathrm{CO}$, as condições do trabalho e a saúde mental, a fim de expandir o modelo de estresse demanda/ controle de Karasek e Theörell (1990), dado que propõem captar a influência mais ampla do contexto organizacional.

Recentemente, foi publicada por Aguiar, Silva, Carvalho, Ferreira e Jesus (2017) uma revisão da literatura nacional, abrangendo os anos de 2000 a 2015 , sobre as relações entre CO, estresse ocupacional e burnout. Quanto ao estresse ocupacional, foram incluídos na revisão somente cinco trabalhos empíricos, o que demonstrou, segundo os autores, a escassez de estudos brasileiros sobre o tema.

A primeira pesquisa empírica da revisão citada anteriormente foi a de Paschoal e Tamayo (2005), que investigou entre trabalhadores de uma instituição bancária a influência da interferência família-trabalho e dos valores laborais no estresse ocupacional. Para a análise dos valores organizacionais, utilizaram a Escala de Valores Relativos ao Trabalho, validada por Porto e Tamayo (2003), que investiga os fatores realização no trabalho, relações sociais, prestígio e estabilidade. Para avaliar o estresse ocupacional, utilizaram uma medida geral de estresse, a Escala de Estresse no Trabalho de Paschoal e Tamayo (2004). Os resultados mostraram que somente a variável interferência família-trabalho explicou a variável estresse ocupacional, não encontrando dessa forma relação significativa entre os valores organizacionais e o estresse ocupacional.

Posteriormente, Tamayo (2007) investigou a relação dos valores organizacionais com o estresse no trabalho em trabalhadores de instituições bancárias de agências de São Paulo, Rio de Janeiro e Brasília. Foi utilizada a mesma medida de investigação para o estresse ocupacional que a pesquisa citada previamente, a Escala de Estresse no Trabalho (Paschoal \& Tamayo, 2004). Já os valores organizacionais foram avaliados pelo Inventário de Perfis de Valores Organizacionais (IPVO) de Oliveira e Tamayo (2004), composto pelos valores: autonomia, bem-estar, realização, tradição, conformidade, preocupação com a coletividade, prestígio e domínio. Identificaram, por análise de regressão múltipla, uma solução explicativa de $32,3 \%$ da variância do estresse no trabalho. Assim, quatro dos fatores do IPVO foram preditores do estresse, sendo eles: (i) autonomia - tem o objetivo de oferecer desafios, criatividade e variedade no trabalho; (ii) preocupação com a coletividade - condição da organização em promover a justiça, a honestidade e a igualdade no trabalho; (iii) realização - valorização da empresa em relação à competência e ao sucesso dos trabalhadores; e (iv) conformidade - promoção da correção, boas maneiras no trabalho e respeito às normas da organização. A análise da direção da relação de cada um dos fatores com o estresse revelou que quanto maior a importância conferida pela organização aos valores de autonomia, preocupação com a coletividade e realização, menor será o estresse no trabalho. Somente o fator conformidade com o estresse teve relação positiva, o que indicou que quanto mais a organização prioriza os valores de conformidade, maior será o estresse.

Canova e Porto (2010), em estudo com professores do ensino médio, constataram a influência dos valores organizacionais sobre o nível de estresse ocupacional, também medidos pelos instrumentos IPVO (Oliveira \& Tamayo, 2004) e Escala de Estresse no Trabalho (Paschoal \& Tamayo, 2004). Os resultados indicaram que os valores organizacionais têm impacto no estresse ocupacional, por meio da constatação de que, quanto mais o professor percebe valores organizacionais de autonomia, bem-estar, ética e preocupação com a coletividade, menos ele relata estresse ocupacional.

Lucena (2010), em investigação com docentes universitários de instituições privadas de Guarulhos (SP), encontrou inexistência de relações significativas entre CO e estresse ocupacional. No estudo, a CO foi investigada pelo Questionário de Cultura Organizacional, traduzido e adaptado por Santos (2000), que considera a estrutura organizacional em quatro tipos culturais: cultura grupal, inovativa, racional e hierárquica. Já as condições psicossociais do trabalho que podem levar ao estresse ocupacional foram avaliadas pelo modelo ERI, de Siegrist (1996), que prevê a relação entre esforço no trabaIho (pressão de tempo, interrupções, responsabilidades, demandas crescentes, pressão na atividade) e recompensas (gratificação monetária, respeito/apoio no trabalho e segurança/oportunidade na carreira).

Feitosa, Belo e Silva (2014) investigaram a influência da CO nos níveis de estresse ocupacional em funcionários de um hospital público do município de Parnaíba (PI). A CO foi medida pelo Instrumento Brasileiro para a Análise da Cultura Organizacional (IBACO), de Ferreira, Assmar, Estol, Helena e Cisne (2002), que avalia práticas e valores organizacionais. Os níveis de estresse foram avaliados pela Escala de Estresse no Trabalho de Paschoal e Tamayo (2004). Constatou-se correlação fraca, nesse sentido, apesar de existir variação concomitante entre as duas variáveis, não sendo possível identificar relação de causa-efeito.

Ao analisar essas pesquisas, identificam-se inconsistências entre os resultados, devido, em parte, à utilização de diferentes instrumentos para analisar o mesmo fenômeno, a exemplo de algumas pesquisas terem incluído somente o valor organizacional como medida de avaliação na $\mathrm{CO}$. Assim, o presente estudo pretende contribuir para o entendimento das relações entre $\mathrm{CO}$ e o risco para o estresse ocupacional, particularmente no contexto de universidade pública federal, não apenas por um dos elementos que compõem a $\mathrm{CO}$, mas sim por meio do modelo de Hofstede, que contempla tanto os valores organizacionais quanto as práticas organizacionais.

Para o estudo do risco para o estresse ocupacional, priorizou-se o modelo ERI, reconhecido por medir o risco psicossocial relacionado ao trabalho, supondo que seus efeitos positivos, como a disponibilidade de ser reconhecido e estimado, ter salário adequado e estrutura de oportunidade social, atuam como um pré-requisito de troca (reciprocidade) na vida social a partir dos esforços e dedicação ao trabalho (Siegrist, 1996). Desse modo, considera-se enquanto hipótese de pesquisa que a CO exerce impacto no desequilíbrio entre esforço-recompensa no trabalho.

\section{Método}

Trata-se de um estudo exploratório-descritivo, de abordagem quantitativa e de corte transversal. Em virtude que apresentou interesse em descrever características da população de servidores técnico-administrativos do ambiente universitário, em um recorte único e limitado no tempo, por meio da quantificação tanto na coleta de informações quanto no tratamento dos dados realizada por técnicas estatísticas, a fim de descobrir e classificar a relação e influência entre as variáveis. A análise baseou-se no modelo teórico-metodológico do Work Stress (Seligmann-Silva, 1995; Jacques, 2003), com inclusão de aspectos sociais referentes ao âmbito da organização de trabalho e pela busca da interação dinâmica entre a pessoa e o seu ambiente de trabalho (Glina, 2014).

\section{Participantes}

Participaram do estudo servidores técnico-administrativos de uma universidade federal do Estado do Mato Grosso do Sul, Brasil. A partir de um universo de $\mathrm{N}=1026$ servidores ativos, foi calculado o número amostral aleatório simples, para que todos tivessem a mesma probabilidade de fazer parte do estudo, estabelecido um 
intervalo de confiabilidade de $95 \%$, com margem de erro ou erro máximo admitido em $5 \%$, que resultou em $\mathrm{N}=280$ trabalhadores. Em virtude de possíveis perdas de informações devido a formulários incompletos, não aceite de participação na pesquisa ou, ainda, do não acesso ao servidor, após três visitas ao local de trabalho, estabeleceu-se um acréscimo de 70 indivíduos (25\%), ficando a amostra final pós-coleta composta por $\mathrm{N}=302$ servidores.

Os critérios de inclusão foram: (i) servidores da carreira técnico-administrativa; (ii) concursados; e (iii) ativos. Os critérios de exclusão foram: (i) servidores que não aceitaram participar da pesquisa; (ii) servidores que estavam de atestado médico, licenças ou férias; (iii) funcionários terceirizados; (iv) servidores do hospital universitário (NHU); (v) servidores que estavam há menos de 6 meses na universidade; (vi) servidores com deficiência visual, tendo em vista que os instrumentos foram autoaplicáveis; e (vii) servidores não encontrados após três visitas ao local de trabalho.

\section{Procedimentos}

Inicialmente, foi feito um estudo-piloto com 35 servidores técnico-administrativos do ambiente universitário, em exercício em um campus de uma universidade situada no interior de Mato Grosso do Sul, Brasil. Realizado em 27 e 28 de junho de 2016, objetivou adequar os instrumentos de pesquisa e conhecer o tempo necessário para a aplicação dos questionários. Posteriormente, para a realização do trabalho de campo da pesquisa, os instrumentos foram administrados de forma presencial e autopreenchível pelos participantes, em seus locais de trabalho. 0 tempo médio de duração foi de 40 minutos e ocorreu entre 22 de julho a 31 de outubro de 2016.

O estudo seguiu todas as normas éticas da Resolução no 466 do Conselho Nacional de Saúde (CNS) (Brasil, 2012), obtendo-se autorização das instâncias competentes e aceite de todos os envolvidos na pesquisa de forma voluntária e autorizada por meio do Termo de Consentimento Livre e Esclarecido (TCLE), aprovado pelo Comitê de Ética em Pesquisa da Universidade Católica Dom Bosco (UCDB), em 5 de novembro de 2015, sob o no 50167215.5.0000.5162.

\section{Instrumentos}

Os instrumentos utilizados foram: (i) Desequilíbrio entre Esforço-Recompensa no Trabalho (Effort-Reward Imbalance Questionnaire - ERI); e (ii) Instrumento Brasileiro para Avaliação da Cultura Organizacional (IBACO).

O ERI foi utilizado para mensurar os fatores psicossociais do ambiente de trabalho, considerando o desequilíbrio entre o alto esforço dispensado pelo trabalhador e a baixa recompensa recebida no trabalho. O ERI foi desenvolvido por Siegrist (1996) e, em sua versão longa, era composto por 46 itens. Posteriormente, para facilitar a aplicação em estudos epidemiológicos, Siegrist et al. (2004) elaboraram uma versão curta contendo 23 itens. O ERI foi traduzido, adaptado e validado para o português brasileiro por Chor, Werneck, Faerstein, Alves e Rotenberg (2008) e também por Silva e Barreto (2010) em sua versão curta.

A versão utilizada pelo presente estudo foi a de Silva e Barreto (2010), devido à facilidade de acesso e a fidelidade ao instrumento original. O ERI é organizado em três escalas unidimensionais: esforço (6 itens), recompensa (11 itens) e supercomprometimento (6 itens), com boa confiabilidade da escala pela variação de consistência interna (alfa de Cronbach) 0,70, 0,95 e 0,86, respectivamente. A escala de recompensa é subdividida em três subescalas: reconhecimento, segurança no trabalho e promoção no emprego/salário. Nas escalas de esforço e recompensa, as respostas variaram no grau de concordância ou discordância, com escores de 1 a 5. Na escala de supercomprometimento, as respostas variaram entre discordar fortemente e concordar fortemente, com escores entre 1 e 4 .

Destacam-se alguns estudos realizados em contextos do serviço público que utilizaram esse instrumento, em avaliação de diferentes desfechos da saúde: doença cardiovascular em funcionários públicos britânicos (Bosma, Siegrist \& Marmo, 1998), estado de saúde autorreferido em enfermeiras brasileiras (Griep et al., 2011), ansiedade e depressão no trabalho de funcionários de ensino superior do Reino Unido (Mark \& Smith, 2012) e sofrimento psicológico entre trabalhadores administrativos de organizações públicas do Canadá (Ndjaboue, Brisson, Talbot \& Vézina, 2017).

Para mensurar os fatores predominantes da $\mathrm{CO}$ da universidade pesquisada, foi utilizado o IBACO, construído e validado por Ferreira et al. (2002). O instrumento, em uma revisão sistemática da literatura realizada por Barale e Santos (2017), foi o mais aplicado em âmbito nacional desde sua validação. Entre estudos desenvolvidos com servidores públicos e que utilizaram o IBACO, destacam-se as pesquisas realizadas em ambiente universitário (Souza, Alves, Vizzoto, Tavares \& Moreira, 2013) e contexto hospitalar (Carvalho, Rocha, Marziale, Gabriel \& Bernardes, 2013; Rocha, Marziele, Carvalho, Id \& Campos, 2014; Rocha, Gaioli, Camelo, Mininel \& Vegro, 2016).

A versão aplicada do IBACO foi a reduzida, de 30 itens, composta pelos fatores: valores de profissionalismo cooperativo (PC) $(\alpha=0,87)$; valores de profissionalismo competitivo (PCI) $(\alpha=0,76)$; valores de satisfação e bem-estar dos empregados (SBE) $(\alpha=0,88)$; práticas de integração externa (IE) $(\alpha=0,85)$; prática de recompensa e treinamento (RT) $(\alpha=0,80)$; e prática de promoção do relacionamento interpessoal (PRI) $(\alpha=0,71)$ (Quadro 1). O IBACO conta com uma escala tipo Likert, em função do grau em que o conteúdo de cada afirmativa aplica-se à organização, que varia entre: (1) não se aplica de modo nenhum; (2) pouco se aplica; (3) aplica-se razoavelmente; (4) aplica-se bastante; e (5) aplica-se totalmente.

Tabela 1

Descrição dos fatores relativos aos valores e práticas organizacionais do IBACO

Valorização dos empregados que executam suas tarefas com eficácia Profissionalismo e competência, espírito de colacooperativo boração, habilidade, dedicação, iniciativa para o alcance das metas organizacionais.

Fatores relativos Profissionalismo Valorização da competência, do dea competitivo sempenho e da eficácia individuais, valores ainda que seja necessário "passar por cima" do colega.

Satisfação e bem- Valorização do bem-estar, da satis-estar dos empre- fação, da motivação dos empregagados dos e da humanização do local de trabalho, para torná-lo agradável e prazeroso.

Fatores relativos Integração externa Práticas voltadas para o planejaàs práticas mento estratégico, a tomada de decisão e o atendimento ao cliente externo.

Recompensa e Práticas vinculadas aos clientes treinamento internos e aos sistemas de recompensa e treinamento adotados pela empresa.

Promoção do rela- Práticas orientadas para a promocionamento inter- ção das relações interpessoais e pessoal satisfação dos empregados para favorecer a coesão interna.

Fonte: elaborado com base em Ferreira e Assmar (2008). 


\section{Tratamento estatístico e análise}

Para o tratamento estatístico dos dados, recorreu-se ao software $R$ for Statistical Computing, na versão estável 3.2.5, de abril de 2016. Conforme Siegrist et al. (2004), a análise do ERI foi realizada pela razão esforço-recompensa (razão ER), elaborada por meio da fórmula: e/( $\left.r^{*} c\right)$, em que "e" é a soma do escore obtido pelas perguntas de esforço; " $r$ " é o escore obtido pela soma das perguntas de recompensa; e "c" é um fator de correção $(0,545454)$. Considerase o número de itens divergentes entre as dimensões de esforço (numerador) e recompensa (denominador), na ordem de 6 para 11. A razão ER foi categorizada em tercis e dicotomizada; assim, os dois tercis inferiores indicaram "equilíbrio" entre esforço-recompensa, e o tercil superior indicou "desequilíbrio" entre esforço-recompensa.

A análise do IBACO, de acordo com Ferreira e Assmar (2008), foi efetuada mediante o cálculo da soma dos pontos atribuídos a cada um dos itens que integram o fator e de sua divisão pelo número de itens que o compõem. Posteriormente, calculou-se a média dos escores atribuídos ao fator por todos os participantes, a fim de obter o escore médio da organização como um todo.

As análises dos dados entre o ERI e IBACO foram realizadas mediante o teste de diferença entre médias ( $t$-Student) para investigar possíveis diferenças da manifestação dos fatores do IBACO entre os grupos em equilíbrio e desequilíbrio esforço-recompensa no trabaIho. Posteriormente, para investigar a hipótese da pesquisa, H1: a CO exerce impacto no desequilíbrio entre esforço-recompensa no trabalho, análises de regressão logística binária foram conduzidas para identificar a predição dos fatores do IBACO para ocorrência do ERI. Adotou-se nível de significância de $5 \%(p \leq 0,05)$.

\section{Resultados}

Os resultados do ERI, analisado pela razão ER (teste de uma proporção), identificaram a maior parte dos servidores $(68,2 \%)$ em equilíbrio esforço-recompensa e $(31,8 \%)$ de participantes "expostos" ao desequilíbrio esforço-recompensa. Destaca-se que não foram apresentadas análises da escala de supercomprometimento do instrumento ERI, devido opção dos pesquisadores de aprofundarem neste estudo o impacto da CO somente no desequilíbrio entre esforço-recompensa no trabalho.

$\mathrm{Na}$ análise da CO pelo IBACO, os fatores identificados com médias em ordem decrescente foram: integração externa - IE (média = 3,3); promoção do relacionamento interpessoal - PRI (média $=3,3$ ); profissionalismo cooperativo - PC (média $=3,1$ ); satisfação e bem-estar-SBE (média = 2,6); recompensa e treinamento - RT (média = $2,5)$; e profissionalismo competitivo - $\mathrm{PCl}$ (média $=2,1$ ). Ressalta-se que médias classificadas na variação 3 indicam, no nível da escala, percepção de "aplica-se razoavelmente", já variação 2, "pouco se aplica".

Os resultados do IBACO e ERI (grupo de servidores "expostos" ao desequilíbrio esforço-recompensa no trabalho) foram analisados pelo teste $t$-Student (Tabela 2). Identificou-se que os valores e as práticas organizacionais, com exceção do valor $\mathrm{PCl}$, apresentaram diferença significativa entre as médias. Isso mostra que os servidores que se encontram expostos ao desequilíbrio entre esforço-recompensa indicaram menores médias nos fatores do IBACO.

A análise de regressão logística, calculada para o ERI (Tabela 3), mostrou que $42,9 \%$ da variância desse modelo foi explicada pelo fator satisfação e bem-estar da CO. Assim, deve-se observar que, ao considerar o grupo dessas variáveis preditoras, apenas um fator da CO (satisfação e bem-estar) contribuiu significativamente para a variância explicada.
Tabela 2

Relação entre os instrumentos IBACO e ERI

\begin{tabular}{|c|c|c|c|c|c|c|c|}
\hline \multirow{2}{*}{$\begin{array}{c}\text { Valores e } \\
\text { práticas } \\
\text { organiza- } \\
\text { cionais }\end{array}$} & \multicolumn{3}{|c|}{ Equilíbrio } & \multicolumn{3}{|c|}{ Desequilíbrio } & \multirow[b]{2}{*}{$\mathrm{p}$-valor } \\
\hline & Média & $\begin{array}{l}\text { Me- } \\
\text { diana }\end{array}$ & $\begin{array}{l}\text { Desvio- } \\
\text {-padrão }\end{array}$ & $\begin{array}{l}\text { Mé- } \\
\text { dia }\end{array}$ & $\begin{array}{l}\text { Media- } \\
\text { na }\end{array}$ & $\begin{array}{l}\text { Desvio- } \\
\text {-padrão }\end{array}$ & \\
\hline PC & 3,3 & 3,2 & 0,80 & 2,8 & 2,8 & 0,69 & $<0,00001$ \\
\hline $\mathrm{PCl}$ & 2,2 & 2,2 & 0,72 & 2,0 & 2,0 & 0,65 & 0,0178 \\
\hline SBE & 2,8 & 2,6 & 0,82 & 2,2 & 2,2 & 0,67 & $<0,00001$ \\
\hline IE & 3,5 & 3,4 & 0,78 & 3,1 & 3,0 & 0,71 & $<0,00001$ \\
\hline RT & 2,6 & 2,6 & 0,75 & 2,1 & 2,0 & 0,53 & $<0,00001$ \\
\hline PRI & 3,4 & 3,4 & 0,69 & 3,0 & 3,0 & 0,74 & $<0,00001$ \\
\hline
\end{tabular}

Notas: $\mathrm{PC}=$ Profissionalismo cooperativo; $\mathrm{PCl}=$ Profissionalismo competitivo; $\mathrm{SBE}=$ Satisfação e bem-estar; IE = Integração externa; RT = Recompensa e treinamento; PRI = Promoção do relacionamento interpessoal.

Tabela 3

Análise dos fatores do IBACO na predição do modelo ERI por regressão logística

\begin{tabular}{llll}
\hline \multicolumn{1}{c}{ Fatores } & \multicolumn{2}{c}{ ERI (variável dependente) } \\
\cline { 2 - 4 } \multicolumn{1}{c}{ IBACO } & Correlação & Coeficientes (B) & Odds Ratio \\
\hline PC & $-0,257$ & 0,0138 & 1,01 \\
PCI & $-0,140$ & 0,2672 & 1,31 \\
SBE & $-0,339$ & $-0,7002 *$ & 0,50 \\
IE & $-0,249$ & $-0,1162$ & 0,89 \\
RT & $-0,311$ & $-0,5780$ & 0,56 \\
PRI & $-0,235$ & $-0,0976$ & 0,91 \\
& & r2 & 0,505 \\
& & r2 (ajust) & 0,429 \\
\hline
\end{tabular}

Notas: $\mathrm{PC}=$ Profissionalismo cooperativo; $\mathrm{PCl}=$ Profissionalismo competitivo; SBE = Satisfação e bem-estar; IE = Integração externa; RT = Recompensa e treinamento; $\mathrm{PRI}=$ Promoção do relacionamento interpessoal.

${ }^{*} p<0,05$.

\section{Discussão}

A prevalência de $31,8 \%$ de participantes em risco para o ERI está na média prevista por Siegrist (2001), de que 10 a $40 \%$ da força de trabalho experimentam algum grau de desequilíbrio entre esforço-recompensa. Entretanto, ressalta-se que, para além de médias comparativas e previstas entre os diferentes grupos ocupacionais, essa prevalência sinaliza que estes servidores estão em risco para adoecimento e com possíveis perdas de sua capacidade laboral.

Em relação ao IBACO, o principal fator de valor organizacional percebido pelos servidores técnico-administrativos foi o do profissionalismo cooperativo (PC) (média $=3,1$ ), o que indica, no nível da escala "aplica-se razoavelmente", a valorização de trabalhadores que executam tarefas com eficiência e competência, além de demonstrarem espírito de colaboração, dedicação, profissionalismo e capacidade de iniciativa para o alcance das metas da organização. Já as práticas organizacionais mais identificadas no IBACO foram integração externa (IE) e promoção do relacionamento interpessoal (PRI), que apresentaram a média de 3,3, situando-se no nível da escala "aplica-se razoavelmente". A análise desses dados sugere que tais fatores contribuem para o alcance das metas comuns da organização, que são o atendimento do público externo (alunos), além de serem encontrados indicativos de uma cultura amparada em um bom desenvolvimento do trabalho em equipe.

A análise da relação entre $\mathrm{CO}$ e risco para estresse ocupacional, por meio do teste $t$-Student, encontrou que os valores e as práticas organizacionais de profissionalismo cooperativo (PC), satisfação e bem-estar (SBE), integração externa (IE), recompensa e treinamento (RT) e promoção do relacionamento interpessoal (PRI) apresentaram, significativamente, menores médias no grupo de servidores 
expostos ao desequilíbrio entre esforço-recompensa. Esses dados sinalizam que servidores que se encontravam em desequilíbrio com alto esforço (pressões no trabalho) e baixa recompensa (salário, segurança e reconhecimento no trabalho) perceberam os valores e práticas da CO da instituição, com exceção do valor de profissionalismo competitivo, de forma mais negativa do que o grupo não exposto ao ERI.

Após essa fase preliminar de análise, procedeu-se a testagem do modelo explicativo proposto por meio da regressão logística. Os resultados encontrados confirmaram $\mathrm{H} 1$, ou seja, a $\mathrm{CO}$ exerce impacto no desequilíbrio entre esforço-recompensa, demonstrando que $42,9 \%$ da variância do modelo ERI foi explicada pelo fator satisfação e bem-estar da CO. Destaca-se que, entre os grupos das variáveis preditores, apenas o fator da CO (satisfação e bem-estar) contribuiu significativamente para a variância explicada, havendo correlação significativa negativa, ou seja, quanto menor é a média do fator de satisfação e bem-estar da $\mathrm{CO}$, maior é a exposição ao ERI.

Esse resultado preditor da CO sobre o risco para estresse ocupacional não foi passível de ser comparado com grupo ocupacional semelhante, devido a sua inexistência na literatura, nem com outros grupos ocupacionais que tivessem utilizado os dois instrumentos de medida concomitantemente (IBACO e ERI). Porém, ao comparar com outras pesquisas que utilizaram diferentes medidas de investigação, o estudo de Tamayo (2007) fortaleceu os resultados encontrados, ao constatar que $32,3 \%$ da variância do estresse no trabalho foi explicada pelos valores organizacionais (autonomia, preocupação com a coletividade, realização e conformidade). Também, Canova e Porto (2010), em estudo com professores, identificaram a influência dos valores organizacionais sobre o nível de estresse ocupacional, sugerindo que, quanto mais se percebe valores organizacionais de autonomia, bem-estar, ética e preocupação com a coletividade, menos se encontra o estresse ocupacional.

Esses resultados de predição da CO no estresse ocupacional contradizem outros estudos (Paschoal \& Tamayo, 2005; Lucena, 2010; Feitosa, Belo \& Silva, 2014), provavelmente em razão dos diferentes instrumentos e análises estatísticas utilizados. Nesse sentido, foi considerada adequada a utilização do IBACO e ERI para averiguar a relação entre $\mathrm{CO}$ e risco para estresse ocupacional, devido ao conteúdo teórico que cada instrumento se propõe a investigar, como também à sua robustez psicométrica.

O resultado obtido para a satisfação e bem-estar dos empregados (SBE), fator preditor na análise de regressão, foi de que quanto menor é a média do fator satisfação e bem-estar, maior é a exposição ao desequilíbrio entre esforço-recompensa no trabalho. Este dado confirma a teoria de Ferreira e Assmar (2004), de que quando valores organizacionais deixam de lado a preocupação com o trabalhador, potencialmente surgem conflitos e, por consequência, problemas de saúde. Nesse sentido, é importante proporcionar ambientes organizacionais mais humanizados e que valorizem o bem-estar de seus servidores, uma vez que para promoção da saúde deve-se envolver a inter-relação entre os indivíduos e seus contextos organizacionais e sociais.

Mesmo respondendo somente a uma parte explicativa do fenômeno estudado, a confirmação do valor preditivo do fator satisfação e bem-estar da CO sobre o risco para estresse ocupacional ratificou a crítica da teorização sobre as causas de o estresse ocupacional centrarem-se exclusivamente na culpabilização do sujeito (Puente-Palacios, Pacheco \& Severino, 2013). Assim, confirma-se a necessidade de considerar o contexto organizacional, permeado pela $\mathrm{CO}$, como uma das variáveis relacionadas ao adoecimento mental do trabalhador.
A constatação do impacto do fator satisfação e bem-estar da CO sobre o risco para o estresse ocupacional reforça a importância de gestores e trabalhadores identificarem a CO predominante da organização, com a intenção de promover sua adaptação aos problemas externos e internos, para subsidiar a construção de políticas e intervenções organizacionais que contribuam efetivamente para a promoção de saúde mental no ambiente laboral. Nesse sentido, os achados desta pesquisa apoiam a discussão teórica, por incluírem, além dos atributos individuais, também os aspectos organizacionais e da própria atividade laboral entre os fatores desencadeantes dos efeitos adversos do estresse ocupacional, particularmente identificado por valores e práticas organizacionais da CO.

Destaca-se a importância de maior valorização da satisfação e bem-estar dos empregados (SBE), para que todos os atores envolvidos no ambiente universitário, entre eles a gestão responsável pelos servidores, promovam relações interpessoais positivas, valorizando suportes organizacionais adequados, de colegas e superiores, além de reconhecimento pessoal e profissional. Essas ações têm como objetivo oportunizar maiores percepções de bem-estar, satisfação e humanização do local de trabalho e, por consequência, maior saúde do trabalhador e da organização. Também é importante manter e fortalecer valores organizacionais permeados pela ética profissional, bem como práticas organizacionais de eficiência e dedicação ao trabalho.

Constatam-se algumas limitações, como o fato de o estudo ser de corte transversal, o que impediu que análises explicativas pudessem ser verificadas ao longo do tempo. Outra restrição foi a possibilidade de ocorrência do "efeito do trabalhador saudável", situação que geralmente minimiza a prevalência de adoecimento, devido ao fato de participantes afastados por licença médica não estarem incluídos na amostra. Também, a limitação da inclusão de apenas um modelo preditor (CO) e uma variável dependente (EO) para a análise do ambiente organizacional. Dessa forma, recomenda-se que futuras pesquisas analisem, tanto em âmbito organizacional quanto individual, outras variáveis potencialmente influenciadoras para a ocorrências do EO, como entrincheiramento organizacional, assédio moral, resiliência do trabalhador e lócus de controle.

Embora esta pesquisa tenha trabalhado com amostragem aleatória, ela está restrita a uma instituição, o que limitou a possibilidade da generalização dos dados a outras instituições públicas. Logo, sugere-se que outros estudos sejam realizados em diferentes universidades públicas, utilizando os instrumentos aplicados nesta pesquisa, com o propósito de possíveis comparações.

\section{Referências}

Aguiar, C. V. N., Silva, E. E. C., Carvalho, B. R., Ferreira, J. C. M., \& Jesus, K. C. O. (2017). Cultura organizacional e adoecimento no trabalho: uma revisão sobre as relações entre cultura, burnout e estresse ocupacional. Revista Psicologia, Diversidade e Saúde, 6(2), 121-131. doi: 10.17267/2317-3394rpds.v6i2.1157

Ansoleaga, E. (2015). Indicadores de Salud Mental asociados a riesgo psicosocial laboral en un hospital público. Revista Médica de Chile, 143, 47-55. Recuperado de http://www.scielo.cl/scielo. php?pid=S0034-98872015000100006\&script=sci_arttext

Barale, R. F., \& Santos, B. R. (2017). Cultura organizacional: revisão sistemática da literatura. Revista Psicologia: organizações e trabalho, v. 17, n. 2, p. 129-136. Recuperado de http://pepsic.bvsalud.org/scielo.php?script=sci_arttext\&pid $=$ S1984-66572017000200008

Biron, C., Brun, J. P., \& Ivers, H. (2008). Extent and sources of occupational stress in university staff. Work, 30(4), 511-522. Recuperado de https://www.ncbi. nlm.nih.gov/pubmed/18725713

Borges, L. de O., Guimaraes, L. A. M., \& Silva, S. S. (2013). Diagnóstico e promoção da saúde psíquica no trabalho. In: Borges, L. O., \& Mourão, L. O trabalho e as organizações: atuações a partir da psicologia (pp. 581-618). Curitiba: Artmed.

Bosma, H., Peter, R., Siegrist, J., \& Marmot, M. (1998). Two Alternative Job Stress Models and the Risk of Coronary Heart Disease. Am J Public Health, 
88(1), 68-74. Recuperado de https://www.ncbi.nlm.nih.gov/pmc/articles/ PMC1508386/

Brasil (2012). Conselho Nacional de Saúde. Resolução n. 466, de 12 de dezembro de 2012. Aprovas as diretrizes e normas regulamentadoras de pesquisa envolvendo seres humanos. Diário oficial [da] República Federativa do Brasil. Brasília, DF, 13 jun.2013. Dispõe sobre a realização de pesquisa em Psicologia com seres humanos. Recuperado de http://bvsms.saude.gov.br/bvs/saudelegis/cns/2013/res0466_12_12_2012.html

Canova, K. R., \& Porto, J. B. (2010). O impacto dos valores organizacionais no estresse ocupacional: um estudo com professores de ensino médio. RAM, Rev. Adm. Mackenzie, 11(5), 4-31. doi: 10.1590/S1678-69712010000500002

Carvalho, M. C., Rocha, F. L. R., Marziale, M. H. P., Gabriel, C. S., \& Bernardes, A. (2013). Valores e práticas de trabalho que caracterizam a cultura organizacional de um hospital público. Texto Contexto Enferm, v. 22, n. 3, p. 746-753. doi: 10.1590/S0104-07072013000300022

Chor, D., Werneck, G. L., Faerstein, E., Alves, M. G. M., \& Rotenberg, L. (2008). The Brazilian version of the effort-reward imbalance questionnaire to assess job stress. Cad. Saúde Pública, Rio de Janeiro, 24(1), 219-224, jan. doi: 10.1590/ S0102-311X2008000100022

Dextras-Gauthier, J., Marchand, A., \& Haines, V. (2012). Organizational culture, work organization conditions, and mental health: a proposed integration. International Journal of Stress Management, 19(2), 81-104. doi: 10.1037/ a0028164

Feitosa, C. M. M., Belo, R. P., \& Silva, C. F. L. S. (2014). A influência da cultura organizacional sobre os níveis de estresse laboral dos técnicos em enfermagem. Perspectivas em Psicologia, 18(1), 75-96. Recuperado de http://www. seer.ufu.br/index.php/perspectivasempsicologia/article/view/28580

Ferreira, M. C., \& Assmar, E. M. L. (2004). Cultura, satisfação e saúde nas organizações. In: Tamayo, A. Cultura e saúde nas organizações. Porto Alegre: Artmed, pp. 102-126.

Ferreira, M. C., \& Assmar, E. M. L. (2008). Cultura organizacional. In: Siqueira, M. M. M. (Org.). Medidas do comportamento organizacional: ferramentas de diagnóstico e de gestão. Porto Alegre: Artmed, p. 125-138.

Ferreira, M. C., Assmar, E. M. L., Estol, K. M. F., Helena, M. C. C. G., \& Cisne, M. C. G. (2002). Desenvolvimento de um instrumento brasileiro para avaliação da cultura organizacional. Estudos de Psicologia, 7(2), 271-280. doi: 10.1590/ S1413-294X2002000200008

Glina, D. M. R. (2014). Modelos Teóricos de estresse e estresse no trabalho e repercussões na saúde do trabalhador. In D. M. Glina \& L. E. Rocha (Orgs.), Saúde Mental no Trabalho: da teoria à prática (pp. 03-30). São Paulo: Roca.

Gradella Júnior, O. (2010). Sofrimento psíquico e trabalho intelectual. Cadernos de Psicologia Social do Trabalho, 13(1), 133-148. Recuperado de http://pepsic. bvsalud.org/scielo.php?script=sci_arttext\&pid=S1516-37172010000100011

Griep, R. H., Rotenberg, L., Landsbergis, P., \& Vasconcellos-Silva, P. R. (2011). Uso combinado de modelos de estresse no trabalho e a saúde auto-referida na enfermagem. Rev Saude Publica, 45(1):145-52, 2011. doi: 10.1590/ S0034-89102011000100017

Herr, R. M., Li, J., Loerbroks, A., Angerer, P., Siegrist, J., \& Fischer, J. E. (2017). Effects and mediators of psychosocial work characteristics on somatic symptoms six years later: Prospective findings from the Mannheim Industrial Cohort Studies (MICS). Journal of psychosomatic research, 98, 27-33. doi: 10.1016/j.jpsychores.2017.05.003.

Hofstede, G., Hofstede, G. J., \& Minkov, M. (2010). Cultures and organizations: software of the mind. Intercultural cooperation and its importance for survival. 3th.ed. London: McGraw-Hill. 279p.

Hofstede, G., Neuijen, B., Ohayv, D. D., \& Sanders, G. (1990). Measuring organizational cultures: a qualitative and quantitative study across twenty cases. Administrative Science Quarterly, 35(2), 286-316. doi: 10.2307/2393392

Jacques, M. G. C. (2003). Abordagens teórico-metodológicas em saúde/doença mental \& trabalho. Psicologia \& Sociedade; 15(1), 97-116, jan./jun.2003. doi: 10.1590/S0102-71822003000100006

Karasek, R., \& Theörell, T. (1990). Healthy work: stress, productivity and the reconstruction of working life. New York: Basic Books.

Knudson, E. M. (2009). La Universidad: Un escenario para transformar la conciencia Social de los profesionales? Repert. med. Cir, 18(1), 29-35. Recuperado de https://revistas.fucsalud.edu.co/index.php/repertorio/article/view/527

Landolt, K., O’Donnell, E., Hazi, A., Dragano, N., \& Wright, B. J. (2017). An experimental examination of the effort-reward imbalance model of occupational stress: Increased financial reward is related to reduced stress physiology. Biological psychology, 125, 121-129. doi: 10.1016/j.biopsycho.2017.03.006

Lira, E. R. (2015). La cultura organizacional: caracterización de las MiPyME's del sur de Jalisco. México: Porrúa Print.
López, A. S., González, N. G., Silva, N., Tolfo, S. R., \& Cedeño, A. G. (2015). Atrapados del Goce: trabajo precario em académicos universitarios. In: Godoy, L., \& Ansoleaga, E. Un campo en tensión o tensión entre campos: psicología de las organizaciones y del trabajo en iberoamérica. Santiago, Chile: RIL editores, pp. 375-388.

Lucena, E. S. (2010). Cultura organizacional e estresse ocupacional: um estudo com docentes das instituições privadas de ensino superior do município de Guarulhos. Dissertação de Mestrado em gestão e desenvolvimento regional do departamento de economia, contabilidade e administração. Universidade Taubaté, São Paulo, Brasil. 149p. Recuperado de http://www.bdtd.unitau.br/ tedesimplificado/tde_arquivos/2/TDE-2012-11-20T095340Z-462/Publico/ Eduardo\%20Soares\%20Lucena.pdf

Mark, G., \& Smith, A. P. (2012). Effects of occupational stress, job characteristics, coping, and attributional style on the mental health and job satisfaction of university employees. Anxiety Stress Coping, 25(1), 63-78. doi: 10.1080/10615806.2010.548088

Ndjaboue, R., Brisson, C., Talbot, D., \& Vézina, M. (2017). Chronic exposure to adverse psychosocial work factors and high psychological distress among white-collar workers: A 5-year prospective study. Journal of psychosomatic research, 94, 56-63. doi: 10.1016/j.jpsychores.2017.01.001.

Oliveira, A. F., \& Tamayo, A. (2004). Inventário de perfis de valores organizacionais. Revista de Administração USP, 39(2), 129-149. Recuperado de http://www.spell.org.br/documentos/ver/16788/inventario-de-perfis-devalores-organizacionais---

Paschoal, T. \& Tamayo, A. (2004). Validação da Escala de Estresse no Trabalho. Estudos de Psicologia, 9(1), 45-52. doi: 10.1590/\$1413-294X2004000100006

Paschoal, T., \& Tamayo, A. (2005). Impacto dos valores laborais e da interferência família-trabalho no estresse ocupacional. Psicologia: Teoria e Pesquisa, 21 (2), 173-180. doi: 10.1590/S0102-37722005000200007

Peterson, M., \& Wilson, J. F. (2002). The culture-work-health model and work stress. Am J Health Behav, 26 (1), 16-24. Recuperado de https://www.ncbi. nlm.nih.gov/pubmed/11795601

Porto, J. B. \& Tamayo, A. (2003). Escala de valores relativos ao trabaIho - EVT. Psicologia: Teoria e Pesquisa, 19(2), 45-152. doi: 10.1590/ S0102-37722003000200006

Puente-Palacios, K. E., Pacheco, E. A., \& Severino, A. F. (2013). Clima organizacional e estresse em equipes de trabalho. Revista Psicologia: Organizações e Trabalho, 13(1), 37-48. Recuperado de http://pepsic.bvsalud.org/scielo. php?script=sci_arttext\&pid=S1984-66572013000100004

Rocha, F. L. R., Marziale, M. H. P., Carvalho, M. C., Id, S. F. C., \& Campos, M. C. T. (2014). A cultura organizacional de um hospital público brasileiro. Rev EsC Enferm USP, 48(2), p. 308-314. doi: 10.1590/S0080-623420140000200016

Rocha, F. L. R., Gaioli, C. C. L. O., Camelo, S. H. H., Mininel, V. A., \& Vegro, T. C. (2016). Cultura organizacional de um hospital psiquiátrico e resiliência dos trabalhadores de enfermagem. Revista brasileira de enfermagem, 69(5), p. 817-824. doi: 10.1590/0034-7167.2016690501

Santos, N. M. B. F. (2000). Cultura organizacional e desempenho: pesquisa, teoria e aplicação. Lorena: Stiliano.

Seligmann-Silva, E. (1995). Psicopatologia e saúde mental no trabalho. In R. Mendes (Org.), Patologia do trabalho (pp. 287-310). Rio de Janeiro: Atheneu.

Siegrist, J. (1996). Adverse health effects of high-effort/low-reward conditions. Journal of Occupational Health Psychology, 1(1), 27-41. doi: 10.1037/1076-8998.1.1.27

Siegrist, J. (2001). A theory of occupational stress. In J. Dunham (Ed.). Stress in the workplace: past, present and futures (pp. 52-66). London: Whurr Publishers.

Siegrist, J., Starke, D., Chandola, T., Godin, I., Marmot, M., Niedhammer, I., \& Peter, R. (2004). The measurement of effort-reward imbalance at work: European comparisons. Soc Sci Med. 58(8), 1483-99. doi: 10.1016/ S0277-9536(03)00351-4

Silva, L. S., \& Barreto, S. M. (2010). Adaptação transcultural para o português brasileiro da escala effort-reward imbalance: um estudo com trabalhadores de banco. Rev Panam Salud Publica. 27(1), 32-6. Recuperado de https://scielosp. org/pdf/rpsp/2010.v27n1/32-36/pt

Souza, H. B., Alves, J. N., Vizzoto, A. D., Tavares, C. E. M., \& Moreira, S. L. (2013). Identificação dos traços da cultura organizacional predominante em uma instituição de ensino superior. In: Congresso online de Administração, 2013. Anais... online: CONVIBRA, 2013. Recuperado de http://www.convibra.com. br/upload/paper/2013/34/2013_34_7655.pdf

Tamayo, A. (2007). Impacto dos valores da organização sobre o estresse ocupacional. RAC-eletrônica, 1(2), 20-33. Recuperado de http://www.anpad.org.br/ periodicos/arq_pdf/a_633.pdf

Tamayo, A. (2008). Valores organizacionais e estresse no trabalho. In: Tamayo, A. (Org.). Estresse e cultura organizacional. São Paulo: Casa do Psicólogo, pp. 333-379. 
Venables, K. M., \& Allender, S. (2006). Occupational health needs of universities: a review with an emphasis on the United Kingdom. Occup Environ Med, 63, 159-167. doi: 10.1136/oem.2005.022145 